\title{
Impacto das mídias sociais nas ações de educação em saúde voltadas à população
}

\author{
Impact of social media on health education actions for the population \\ Impacto de las redes sociales en las acciones de educación sanitaria para la población
}

\author{
Maria Andressa Gomes de Lima \\ ORCID: https://orcid.org/0000-0002-0543-4399 \\ Centro Universitário INTA, Brasil \\ E-mail: andressagomesdelima47@gmail.com \\ Lívia Sayuri Félix Mendes \\ ORCID: https://orcid.org/0000-0001-7166-7708 \\ Centro Universitário INTA, Brasil \\ E-mail: livya.mendessp@gmail.com \\ Ana Luiza Linhares Beserra Machado \\ ORCID: https://orcid.org/0000-0002-7585-9739 \\ Faculdade Princesa do Oeste, Brasil \\ E-mail: analuizabeserra@hotmail.com \\ Milena Cordeiro de Freitas \\ ORCID: https://orcid.org/0000-0003-0208-9400 \\ Faculdade Cearense, Brasil \\ E-mail: barra.milenafreitas@hotmail.com \\ Thaisnara Rocha dos Santos \\ ORCID: https://orcid.org/0000-0002-3855-1866 \\ Universidade Estadual do Ceará, Brasil \\ E-mail: tnara97@gmail.com \\ Antônio Diego Costa Bezerra \\ ORCID: https://orcid.org/0000-0002-2441-2961 \\ Centro Universitário Unifanor, Brasil \\ Fundação Oswaldo Cruz, Brasil \\ E-mail: diegocostamjc@gmail.com \\ Francisco Taynãn Balbino Gomes \\ ORCID: https://orcid.org/0000-0003-2884-7417 \\ Centro Universitário Inta, Brasil \\ E-mail: gomestaynangomes@hotmail.com \\ Karine da Cruz da Silva Feitosa \\ ORCID: https://orcid.org/0000-0002-5113-8670 \\ Centro Universitário Presidente Antônio Carlos, Brasil \\ E-mail: krafaela444.kr@gmail.com \\ Cidianna Emanuelly Melo do Nascimento \\ ORCID: https://orcid.org/0000-0001-5477-4413 \\ Universidade Estadual do Ceará, Brasil \\ E-mail: profa.cidianna.melo@gmail.com \\ Maria Eduarda Almeida Marçal \\ ORCID: https://orcid.org/0000-0002-7976-7487 \\ Instituto Federal de Educação, Ciência e Tecnologia de Pernambuco, Brasil \\ E-mail: Eduarda.marcal5@gmail.com \\ Victoria Caroline da Silva \\ ORCID: https://orcid.org/0000-0002-7091-6749 \\ Centro Universitário Católica de Quixadá, Brasil \\ E-mail: vicctoriacsilva@outlook.com \\ Luciano Santos da Silva Filho \\ ORCID: https://orcid.org/0000-0003-2325-3716 \\ Escola de Saúde Pública do Ceará, Brasil \\ E-mail: lucianofilhofisio@gmail.com
}

\section{Resumo}

Objetivos: Analisar o impacto das mídias sociais para a educação em saúde para a população. Metodologia: Trata-se de uma revisão integrativa de literatura, realizada no período de outubro a dezembro de 2020, seguindo as seis etapas indicadas para a revisão integrativa, a pergunta norteadora foi definida com base no acrônimo PICo. Utilizou-se as bases de dados: Literatura Latino Americana e do Caribe em Ciências da Saúde (LILACS), Medical Literature Analysis and Retrieval System Online (MEDLINE) via PubMed, Base de Dados de Enfermagem (BDENF) e Índice Bibliográfico Español en Ciencias de la Salud. Resultados: Os estudos revelam que as Tecnologias de Informação e Comunicação (TIC), têm ganhado cada vez mais adesão e crédito na proliferação de conteúdo, tendo em vista a 
acessibilidade de grande parte da população, gerando valorização e desenvolvimento de metodologias inovadoras. Também se tornando bastante importante no âmbito acadêmico, destruindo barreiras, promovendo a propagação do conhecimento. Considerações finais: Com base nos resultados, observa-se que o uso da tecnologia, e de forma especial das mídias digitais, para a inserção da população na construção do seu próprio conhecimento tem grande valia e credibilidade, pois dessa forma, as informações, podem ser repassadas de maneira mais rápida e eficiente para a população.

Palavras-chave: Mídias sociais; Educação em saúde; Tecnologia.

\begin{abstract}
Objectives: To analyze the impact of social media for health education for the population. Methodology: This is an integrative literature review, carried out from October to December 2020, following the six steps indicated for the integrative review, the guiding question was defined based on the acronym PICo. The following databases were used: Latin American and Caribbean Literature in Health Sciences (LILACS), Medical Literature Analysis and Retrieval System Online (MEDLINE) via PubMed, Nursing Database (BDENF) and Bibliographic Index Español en Ciencias de la Salud. Results: Studies reveal that Information and Communication Technologies (ICT) have been gaining more and more support and credit in the proliferation of content, in view of the accessibility of a large part of the population, generating valuation and development of innovative methodologies. It is also becoming very important in the academic field, destroying barriers, promoting the spread of knowledge. Final considerations: Based on the results, it is observed that the use of technology, and especially of digital media, for the insertion of the population in the construction of their own knowledge has great value and credibility, because in this way, the information can be passed on more quickly and efficiently to the population.
\end{abstract}

Keywords: Social media; Health education; Technology.

\title{
Resumen
}

Objetivos: Analizar el impacto de las redes sociales en la educación para la salud de la población. Metodología: Se trata de una revisión integradora de la literatura, realizada de octubre a diciembre de 2020, siguiendo los seis pasos indicados para la revisión integradora, se definió la pregunta orientadora en base a las siglas PICo. Se utilizaron las siguientes bases de datos: Literatura Latinoamericana y del Caribe en Ciencias de la Salud (LILACS), Sistema de Recuperación y Análisis de Literatura Médica en Línea (MEDLINE) vía PubMed, Base de Datos de Enfermería (BDENF) e Índice Bibliográfico Español en Ciencias de la Salud. Resultados: Los estudios revelan que las Tecnologías de la Información y las Comunicaciones (TIC) han ido ganando cada vez más apoyo y crédito en la proliferación de contenidos, ante la accesibilidad de gran parte de la población, generando valoración y desarrollo de metodologías innovadoras. También se está volviendo muy importante en el ámbito académico, destruyendo barreras, promoviendo la difusión del conocimiento. Consideraciones finales: Con base en los resultados, se observa que el uso de la tecnología, y especialmente de los medios digitales, para la inserción de la población en la construcción de su propio conocimiento tiene un gran valor y credibilidad, pues de esta forma la información puede transmitirse más rápida y eficazmente a la población.

Palabras clave: Redes sociales; Educación en salud; Tecnología.

\section{Introdução}

A educação em saúde é um processo educativo que envolve as relações entre os profissionais de saúde, os gestores que apoiam esses profissionais e a população que necessita construir seus conhecimentos e aumentar sua autonomia nos cuidados individuais e coletivamente. Ademais, visa o desenvolvimento crítico e reflexivo do indivíduo sobre sua saúde, capacitando-o para opinar nas decisões de sua sobre a mesma (Ministério da Saúde, 2006).

A integralidade é um dos princípios do Sistema Único de Saúde (SUS) que trata da atenção integral à saúde em todos os âmbitos do sistema, no qual inclui os saberes, as práticas e as vivências de espaços de cuidados. Por isso, torna-se necessário o desenvolvimento de ações de práticas educativas em saúde pelos profissionais de forma criativa, participativa e que contribuam para o empoderamento dos usuários, melhorando assim processo de saúde-doença (Ministério da Saúde, 2007).

Além disso, contemporaneamente, é possível perceber que há necessidade de complementar o atual modelo de atenção assistencial hegemônico biomédico, o qual é centrado na doença, sendo excessivamente especializado e ainda prioritariamente hospitalar, por um modelo integral, que priorize a promoção da saúde e a prevenção de agravos, e que utilize a educação em saúde de forma participativa e dialógica (Falkenberg, 2014). 
Por esse viés, é notório que a sociedade está passando por momentos de transformações e adaptação na utilização de tecnologias como instrumento de ensino. Válido mencionar que tais mudanças ocorrem devido à globalização e as inovações de tecnologias de informação e comunicação (TICs) que acabam impactando diversas áreas da sociedade, inclusive a educação. Além disso, o conhecimento científico vem se desenvolvendo com o passar do tempo, possibilitando a elaboração de inovações tecnológicas (Cancela, Cancela, Sousa, Silva \& Santos, 2020).

Ademais, conciliar recursos tecnológicos com novas formas de práticas educativas em saúde é uma estratégia que não pode ser negligenciada tanto por quem trabalha em saúde pública quanto por gestores. Embora a aplicação desses recursos tecnológicos na saúde pública seja incipiente, eles já apresentam algumas características, como o olhar voltado não apenas para o cognitivo e para o entretenimento, mas, também, para o gerenciamento e o monitoramento de doenças crônicas, como hipertensão, por exemplo (Morais, Vergara, Brito \& Sampaio, 2020).

Nesse contexto, as mídias sociais têm se apresentado como uma ferramenta poderosa para a disseminação de informações de qualidade, sendo utilizado para trocas de conhecimento, aulas de Educação a Distância (EaD) e consultas online, o que, consequentemente, expande as ferramentas educacionais. Mas vale lembrar que devemos sempre burcas fontes confiáveis e de respaldo, para defrontar informações sem validação cientifica.

Entretanto, isso não é diferente quando se trata da área de saúde, pois diferentes profissionais da área têm utilizado ferramentas do espaço digital como um instrumento para veicular informação. Além disso, a população tende a servir-se desses espaços para buscar informações sobre doenças, expor seus sentimentos e suas experiências com o processo de adoecimento e compartilhar suas angústias e sofrimentos com outros que também estão vivenciando algo parecido. Assim, as ferramentas da web podem ser grandes aliadas nas atividades pedagógicas, tanto na exposição de informações quanto proporcionado espaços colaborativos e interativos entre as pessoas (Cruz, Paulo, Dias, Martins \& Gandolfi, 2011).

Desta forma, o presente estudo visa analisar o impacto das mídias sociais na educação em saúde para a população.

\section{Metodologia}

O presente artigo trata-se de uma revisão integrativa de literatura, realizada no período de outubro a dezembro de 2020, seguindo as seis etapas indicadas para a revisão integrativa, que são: 1-Elaboração da pergunta norteadora; 2-Busca ou amostragem na literatura; 3-Coleta de Dados; 4-Análise crítica dos estudos incluídos; 5-Apresentação dos resultados e 6Discussão dos resultados (Souza; Silva \& Carvalho, 2010).

A pergunta norteadora foi definida com base no acrônimo PICo, conforme recomendado por Araújo (2020), onde definiu-se: P (problema) - impacto das mídias sociais, I (intervenção) - ações de educação em saúde, Co (contexto) - inserção da população. Assim, a pergunta norteadora formulou-se da seguinte maneira: "Quais os impactos das mídias sociais nas ações de educação em saúde voltados à população?

Referente a coleta de dados, utilizou-se as bases de dados: Literatura Latino Americana e do Caribe em Ciências da Saúde (LILACS), Medical Literature Analysis and Retrieval System Online (MEDLINE) via PubMed, Base de Dados de Enfermagem (BDENF) e Índice Bibliográfico Español en Ciencias de la Salud, que foram configuradas para buscar os descritores nos campos "título", "resumo" e "palavras-chave".

Além disso, os descritores controlados considerados para operacionalização da busca foram definidos segundo os Descritores em Ciências da Saúde (DeCS), e utilizados em dois momentos, primeiramente em português, sendo eles: "mídias sociais" "educação em saúde" e "tecnologia", e posteriormente no idioma inglês: "social media", "health education" e "technology", de forma a abranger a literatura nacional e internacional. Os termos foram combinados pelo operador booleano "AND" em ambas as fases de busca. 
Quanto aos critérios de inclusão, determinou-se que os estudos deveriam ter sido publicados nos últimos cinco anos (2016-2020), nos idiomas português, inglês ou espanhol, e estivessem disponíveis na íntegra gratuitamente. Já pelos critérios de exclusão, não foram consideradas as pesquisas que estivessem duplamente indexadas, estudos secundários, literatura cinzenta, e estudos que não respondessem à pergunta norteadora estabelecida, observando-se inicialmente título e resumo.

Ao todo, 14 estudos foram analisados, dos quais 8 atenderam aos objetivos propostos, e foram incluídos no presente estudo. Os artigos selecionados foram lidos na íntegra, e posteriormente sintetizados, em forma de quadro, com suas principais informações, de modo a permitir um panorama geral e possibilitar uma análise crítica.

Por se tratar de um estudo que envolve dados secundários, não houve necessidade de apreciação pelo Comitê de Ética em Pesquisas (CEP).

\section{Resultados e Discussão} em saúde.

O Quadro 1 sintetiza os artigos encontrados que abordam a utilização das redes sociais como instrumento de educação

Quadro 1. Descrição de autores, ano de publicação, título, objetivo e periódico dos artigos encontrados.

\begin{tabular}{|c|c|c|c|}
\hline Autor/ano de publicação & Título & Objetivo & Periódico \\
\hline CRESPO Et al, 2019. & $\begin{array}{l}\text { Modernidade líquida: desafios } \\
\text { para educação em saúde no } \\
\text { contexto das vulnerabilidades } \\
\text { para infecções sexualmente } \\
\text { transmissíveis }\end{array}$ & $\begin{array}{l}\text { compreender os significados que } \\
\text { membros da equipe multiprofissional } \\
\text { de saúde atribuem às tecnologias de } \\
\text { informação e comunicação para } \\
\text { educação em saúde no contexto das } \\
\text { infecções sexualmente transmissíveis } \\
\text { (IST) e Síndrome de } \\
\text { Imunodeficiência Adquirida (AIDS). }\end{array}$ & Rev. enferm. UERJ. \\
\hline $\begin{array}{l}\text { DALMASO e BONAMIGO, } \\
2019 .\end{array}$ & $\begin{array}{l}\text { A pesquisa on-line sobre } \\
\text { amamentação: entre o senso } \\
\text { comum e a OMS na era digital. }\end{array}$ & $\begin{array}{l}\text { Uma vez que a internet assumiu o } \\
\text { papel de fonte preferencial e } \\
\text { espontânea de acesso à informação, o } \\
\text { objetivo deste estudo é sintetizar o } \\
\text { que as mães brasileiras buscam e } \\
\text { encontram na internet sobre } \\
\text { aleitamento materno, por meio de } \\
\text { revisão crítica da literatura científica } \\
\text { dos últimos } 10 \text { anos. }\end{array}$ & $\begin{array}{l}\text { Revista Eletronica de } \\
\text { Comunicação, Informacao } \\
\text { e Inovação em Saúde. }\end{array}$ \\
\hline $\begin{array}{l}\text { FRANÇA, } \quad \text { RABELLO e } \\
\text { MAGNAGO, } 2019 .\end{array}$ & $\begin{array}{l}\text { As mídias e as plataformas } \\
\text { digitais no campo da Educação } \\
\text { Permanente em Saúde: debates } \\
\text { e propostas }\end{array}$ & $\begin{array}{l}\text { Este ensaio buscou debater a } \\
\text { relevância das mídias e das } \\
\text { plataformas digitais como } \\
\text { ferramentas para o desenvolvimento e } \\
\text { gestão de ações de Educação } \\
\text { Permanente em Saúde. }\end{array}$ & Saúde debate. \\
\hline $\begin{array}{l}\text { MONTOYA ACOSTA Et al, } \\
2019 .\end{array}$ & $\begin{array}{l}\text { Teorías pedagógicas que } \\
\text { sustentan el aprendizaje con el } \\
\text { uso de las Tecnologías de la } \\
\text { Información y las } \\
\text { Comunicaciones }\end{array}$ & $\begin{array}{l}\text { Analisar modelos que são utilizados } \\
\text { em processos de aprendizagem com o } \\
\text { uso de informações e comunicações. }\end{array}$ & $\begin{array}{l}\text { Revista información } \\
\text { científica. }\end{array}$ \\
\hline $\begin{array}{l}\text { RAMOS, SOUZA E MELO, } \\
2018 .\end{array}$ & $\begin{array}{l}\text { Incorporação das tecnologias de } \\
\text { informação e comunicação na } \\
\text { integração ensino-serviço dos } \\
\text { cursos de saúde de uma } \\
\text { universidade pública }\end{array}$ & $\begin{array}{l}\text { Com o objetivo de analisar a } \\
\text { incorporação de Tecnologias de } \\
\text { Informação e Comunicação (TICs) } \\
\text { pelos docentes dos nove cursos de } \\
\text { saúde da Universidade Federal de }\end{array}$ & Revista da ABENO. \\
\hline
\end{tabular}




\begin{tabular}{|c|c|c|c|}
\hline & & $\begin{array}{l}\text { Pernambuco (UFPE) nas atividades } \\
\text { de integração ensino-serviço nos } \\
\text { Estágios } \\
\text { Supervisionados } \\
\text { um ECS), realizouse } \\
\text { um estudo observacional e descritivo } \\
\text { com abordagem quantitativa. }\end{array}$ & \\
\hline PAULINO, 2018. & $\begin{array}{l}\text { WhatsApp }{ }^{\circledR} \text { como Recurso } \\
\text { para a Educação em Saúde: } \\
\text { Contextualizando Teoria e } \\
\text { Prática em um Novo Cenário de } \\
\text { Ensino-Aprendizagem }\end{array}$ & $\begin{array}{l}\text { Este relato busca compartilhar uma } \\
\text { experiência bem-sucedida do uso do } \\
\text { aplicativo WhatsApp® como recurso } \\
\text { para a educação médica, destacando o } \\
\text { potencial pedagógico e formativo das } \\
\text { redes sociais, ainda não aproveitado } \\
\text { plenamente pelas universidades. }\end{array}$ & $\begin{array}{lr}\text { Revista } & \text { brasileira de } \\
\text { educação } & \text { médica } \\
\text { (Impresso). } & \end{array}$ \\
\hline BELTRÁN AROCA, 2017. & $\begin{array}{l}\text { ¿Cómo afectan los medios } \\
\text { sociales a la confidencialidad de } \\
\text { los pacientes? Revisión de los } \\
\text { potenciales problemas y } \\
\text { recomendaciones }\end{array}$ & $\begin{array}{l}\text { Os dados pessoais relativos à saúde } \\
\text { do indivíduo são considerados dados } \\
\text { sensíveis, por esse motivo eles } \\
\text { necessitam de uma "proteção } \\
\text { especial". Nos encontramos em uma } \\
\text { sociedade em que as tecnologias de } \\
\text { informação e comunicação (TIC), } \\
\text { especialmente em relação às redes } \\
\text { sociais, oferecem amplas } \\
\text { oportunidades para a troca de } \\
\text { informações médicas, servindo como } \\
\text { apoio, conselho ou assistência aos } \\
\text { colegas que estão na mesma área ou } \\
\text { em qualquer lugar na geografia } \\
\text { mundial. }\end{array}$ & Acta bioethica. \\
\hline SANTOS, 2017. & $\begin{array}{l}\text { Teaching and learning Oral } \\
\text { Radiology via the social } \\
\text { medium WhatsApp }\end{array}$ & $\begin{array}{l}\text { O objetivo desse estudo foi introduzir } \\
\text { a mídia social WhatsApp como uma } \\
\text { ferramenta de ensino no curso de } \\
\text { Radiologia Oral. }\end{array}$ & Revista da ABENO. \\
\hline PINTO E ROCHA, 2016. & $\begin{array}{l}\text { novações na Atenção Primária } \\
\text { em Saúde: o uso de ferramentas } \\
\text { de tecnologia de comunicação e } \\
\text { informação para apoio à gestão } \\
\text { local }\end{array}$ & $\begin{array}{l}\text { O artigo descreve os resultados da } \\
\text { implementação da Rede de } 16 \\
\text { Observatórios de Tecnologias de } \\
\text { Informação e Comunicação em } \\
\text { Serviços de Saúde - Rede OTICS- } \\
\text { RIO - pela Secretaria Municipal de } \\
\text { Saúde do Rio de Janeiro no apoio à } \\
\text { integração da atenção primária, } \\
\text { vigilância e promoção da saúde. }\end{array}$ & $\begin{array}{l}\text { Ciência em saúde } \\
\text { coletiva. }\end{array}$ \\
\hline $\begin{array}{l}\text { Hosam Mamoon Zowawi } \\
\text { Published online } 2015 \text { Oct } 15 . \\
\text { doi: } 10.2196 / \text { jmir.3891 }\end{array}$ & $\begin{array}{l}\text { The Potential Role of Social } \\
\text { Media Platforms in Community } \\
\text { Awareness of Antibiotic Use in } \\
\text { the Gulf Cooperation Council } \\
\text { States: Luxury or Necessity? }\end{array}$ & $\begin{array}{l}\text { Este artigo discute o potencial do uso } \\
\text { de ferramentas de mídia social como } \\
\text { plataformas econômicas e de } \\
\text { educação em massa para aumentar a } \\
\text { conscientização sobre o uso } \\
\text { apropriado de antibióticos no público } \\
\text { em geral e nas comunidades médicas } \\
\text { da Península Arábica }\end{array}$ & J Med Internet Res \\
\hline
\end{tabular}

Fonte: Autores. 
Segundo Crespo, Silva, Costa e Araújo. (2019), a sociedade tem se tornado cada vez mais superficial em suas relações e isso dificulta nas realizações de intervenções de disseminação de conhecimento, com isso faz-se necessário a utilização de ferramentas do cotidiano para propagação de informação.

Os estudos revelam que as Tecnologias de Informação e Comunicação (TIC), têm ganhado cada vez mais adesão e crédito na proliferação de conteúdo, tendo em vista a acessibilidade de grande parte da população. Isso gera valorização do desenvolvimento de metodologias inovadoras, criativas que ajudam no desfecho de comunicação, saúde e comunidade (Pinto \& Rocha, 2016).

De acordo com França, Rabelo e Magnano (2019), as TIC deixam de ser apenas um meio de Educação Permanente em Saúde (EPS) e passa a tomar lugar mais amplo como meio de comando da educação e do serviço em saúde, pois, através delas é possível identificar públicos vulneráveis e trabalhar sobre isso.

Conforme Paulino (2018), o emprego das mídias sociais corrobora com a construção ativa de conhecimento do indivíduo, pois leva ao pensamento crítico de sua conduta e adoção de novos hábitos, sendo por si, um feedback positivo; enfatiza-se que essa abordagem ativa é peça valiosa no aperfeiçoamento do aprendizado.

Dentro do âmbito acadêmico essa ferramenta tem ajudado a romper barreiras, pois é nesse meio em que há construção, desconstrução, iniciação, multiplicação e propagação de conhecimento. Deixando de ser apenas um desenvolvimento industrial que vai na vertical e passando a ser também um crescimento informacional, sendo assim, na horizontal. (Ramos; Souza \& Melo, 2018)

Em contrapartida, Acosta, Castellanos, Arias, Alciva e Ronquillo. (2019) e Santos (2017) expõem que o whatsapp foi inserido como uma TIC, mas enfatizam que apesar levar as informações de forma mais rápida, tende a ter menor adesão; por isso reforçam a necessidade que haja mais investimento de tempo para pesquisar estratégias que melhorem a integração do público com o conteúdo a ser abordado.

De acordo com Aroca e Lopez (2017), ainda existem muitas coisas a serem discutidas uma delas é o sigilo profissional, cujo é princípios éticos. Não é cabível o uso de informações pessoais de terceiros para a divulgação de informações. Por isso, antes de passar qualquer informação adiante é imprescindível o uso correto e a manipulação adequada de dados.

Em síntese, os autores abordam no geral que apesar de as mídias sócias serem uma ferramenta potente, acessível e facilitadora, é necessário ser desenvolvidas mais pesquisas sobre adesão do público, criar meios que limite disseminação de conteúdos inexatos e criar a cultura crítica no leitor, para a busca por conteúdos com vertentes comprovadas.

\section{Conclusão}

Com base nos resultados, observa-se que o uso da tecnologia, e de forma especial das mídias digitais, para a inserção da população na construção do seu próprio conhecimento tem grande valia e credibilidade, visto que por meio destes, as informações, de forma geral, podem ser repassadas de maneira mais rápida e eficiente para a população.

Portanto, deve-se viabilizar o aperfeiçoamento nesta área, para que esse acesso seja de qualidade, compreensível e palpável para todo o público, respeitando todos os princípios que regem a ética e valorizando as diversas ferramentas tecnológicas que estão disponíveis atualmente. Vale salientar que como todos os âmbitos é necessário haver ordem e decência, é fundamental criar políticas públicas em prol de extinguir qualquer sorte de informações inadequadas e fora de validação cientifica.

A título de encerramento, com base em todo conhecimento adquirido até aqui e em concordância com Travieso et.al. (2020), apesar do cenário hostil, o contexto possibilitou novos aprendizados; além de podermos por em prática de forma 
dinâmica e lúdica, as ferramentas que até então eram pouco utilizadas, proporcionando a compreensão da aplicabilidade de tais, no âmbito informacional e educativo.

\section{Referências}

Beltrán Aroca, C., \& Girela López, E. (2017). ¿Cómo afectan los medios sociales a la confidencialidad de los pacientes? Revisión de los potenciales problemas y recomendaciones. Acta Bioethica, 23(1). https://actabioethica.uchile.cl/index.php/AB/article/view/46194/48211.

Cancela, L. B., Cancela, P. A. R. C., de Sousa, L. D., da Silva, D. R., \& dos Santos, A. T. C. (2020). Mapeamento sobre a inserção das tecnologias da informação e comunicação no ensino: estudo de caso das escolas estaduais de carangola/mg. In Anais do Encontro Virtual de Documentação em Software Livre e Congresso Internacional de Linguagem e Tecnologia Online (Vol. 9, No. 1).

Cruz, D. I., Paulo, R. R. D., Dias, W. S., Martins, V. F. \& Gandolfi, P. E. (2011). O uso das mídias digitais na educação em saúde. Disponível em: Cadernos da FUCAMP, 10(13), 130-142/2011.

Falkenberg, M. B., Mendes, T. P. L., Moraes, E. P. \& Souza, E. M. (2014). Educação em saúde e educação na saúde: conceitos e implicações para a saúde coletiva. Disponível em: Ciênc. saúde coletiva .19(3).

Franca, T. Rabello, E. T. \& Magnago, C. As mídias e as plataformas digitais no campo da Educação Permanente em Saúde: debates e propostas. (2019). Saúde debate, 43, 106-115.

Ministério da Saúde. (2006). Secretaria de Gestão do Trabalho e da Educação na Saúde. Brasília, Departamento de Gestão e da Regulação do Trabalho em Saúde. Câmara de Regulação do Trabalho em Saúde. DF: Ministério da Saúde. Brasil.

Ministério da Saúde. (2007). Secretaria de Gestão Estratégica e Participativa. Departamento de Apoio à Gestão Participativa. Caderno de educação popular e saúde. Departamento de Apoio à Gestão Participativa. DF: Ministério da Saúde, 160 p. il. color. Brasil. (Série B. Textos Básicos de Saúde).

Montoya, A., Silva, I. R., Costa, L. S. \& Araújo, I. F. L. (2019). Teorías pedagógicas que sustentan el aprendizaje con el uso de las Tecnologías de la Información y las Comunicaciones. Revista información científica. https://doi.org/10.12957/reuerj.2019.43316.

Morais, E. R., Vergara, C. M. A. C., Brito, F. O. \& Sampaio, H. A. C. Serious games para educação em higiene bucal infantil: uma revisão integrativa e a busca de aplicativos. (2020). Disponível em: Ciênc.saúde coletiva 25 (8).

Paulino, D. B., Martins, C. C. A., Raimondi, G. A. \& Hattori, W. T. (2018). WhatsApp® como Recurso para a Educação em Saúde: Contextualizando Teoria e Prática em um Novo Cenário de Ensino-Aprendizagem. Rev. bras. educ. med., 42(1),. 171-180.

Pinto, L. F. \& Rocha, C. M. F. (2016. Inovações na Atenção Primária em Saúde: o uso de ferramentas de tecnologia de comunicação e informação para apoio à gestão local. Ciênc. saúde coletiva, 1(5), 1433-1448.

Ramos, E. R. L. G., Souza, F. B. \& Melo, M. M. D. C. Incorporação das tecnologias de informação e comunicação na integração ensino-serviço dos cursos de saúde de uma universidade pública. (2018). Revista da ABENO. https://doi.org/10.30979/rev.abeno.v18i3.580.

Santos, G. N. M., Leite, A. F., Figueiredo, P. T. S. \& Melo, N. S. Teaching and learning Oral Radiology via the social medium WhatsApp. (2017).

Souza, M. T. de, Silva, M. D. da, \& Carvalho, R. de. (2010). Revisão integrativa: o que é e como fazer. Einstein (São Paulo), 8(1), 102-106. https://dx.doi.org/10.1590/s1679-45082010rw1134

Travieso., M., Zangara., A., Massini., M., López., M., \& Ivanoff., M.. (2020). \#TecnoEduUNLP en tiempos de aislamiento: oportunidades y tensiones. InterCambios. Dilemas y transiciones de la Educación Superior, 7(2), 119-131. https://dx.doi.org/10.2916/inter.7.2.12

Wánderson Cássio Oliveira Araújo (2020). Recuperação da informação em saúde: construção, modelos e estratégias. ConCl: Conv. Ciênc. Inform, 3(2), 100134, maio/ago. 